\title{
Saturn's internal structure and carbon enrichment
}

\author{
O. Mousis ${ }^{1}$, Y. Alibert ${ }^{2}$, and W. Benz ${ }^{2}$ \\ ${ }^{1}$ Observatoire de Besançon, CNRS - UMR 6091, BP 1615, 25010 Besançon Cedex, France \\ e-mail: 0livier.Mousis@obs-besancon . fr \\ 2 Physikalisches Institut, University of Bern, Sidlerstrasse 5, 3012 Bern, Switzerland
}

Received 19 September 2005 / Accepted 17 November 2005

\section{ABSTRACT}

We use the clathrate hydrate trapping theory to calculate the enrichments in $\mathrm{O}, \mathrm{N}, \mathrm{S}, \mathrm{Xe}, \mathrm{Ar}$ and $\mathrm{Kr}$ compared to solar in Saturn's atmosphere. For this, we calibrate our calculations using two different carbon abundance determinations that cover the domain of measurements published in recent decades: one derived from the NASA Kuiper Airborne Observatory measurements and the other obtained from the Cassini spacecraft observations. We show that these two carbon abundances imply a different minimum heavy element content for Saturn. Using the Kuiper Airborne Observatory measurement for calibration, the amount of ices accreted by Saturn is found to be consistent with current interior models of this planet. On the other hand, using the Cassini measurement for calibration leads to an ice content in the planet's envelope that is higher than the one derived from the interior models. In this case, reconciling the interior models with the amount of $\mathrm{C}$ measured by the Cassini spacecraft requires that significant differential sedimentation of water and volatile species has taken place in Saturn's interior during its lifetime.

Key words. stars: planetary systems - stars: planetary systems: formation - solar system: formation

\section{Introduction}

The standard giant planet formation scenario, namely the socalled core accretion model (Pollack et al. 1996), has been extended by Alibert et al. $(2004,2005 a, b)$ to include migration of the growing planet and proto-planetary disk evolution. These calculations show that the extended core accretion model can lead to the formation of two giant planets closely resembling Jupiter and Saturn in less than 3 Myr (Alibert et al. 2005b, hereafter A05b). Based on these formation models, Alibert et al. (2005c, hereafter A05c) also demonstrated that the volatile enrichments measured in Jupiter's atmosphere can be explained in a way consistent with the constraints set by internal structure modeling (Saumon \& Guillot 2004, hereafter SG04). In this work, we follow the strategy adopted by A05c and utilize the thermodynamical conditions of the protoplanetary disk that led to the formation of Saturn (A05b) to calculate the volatile enrichments in its atmosphere. We then compare the calculated amount of required ices in Saturn with the content of heavy elements predicted by the internal structure models of SG04.

The carbon abundance in Saturn's atmosphere has been the subject of a large number of studies. In our calculations, we adopt two extreme values of $\mathrm{C}$ abundance that cover the domain of measurements published in the last 25 years (see Table 1). From the use of a haze model to derive volume mixing ratios in the near-IR bands, $\mathrm{C}$ has been shown to be enriched by a factor of $3.4 \pm 0.9$ (Kerola et al. 1997; Kuiper Airborne Observatory
(KAO)) in Saturn's atmosphere compared to its solar value ${ }^{1}$ (Lodders 2003; see Table 2). On the other hand, recent Cassini CIRS far-IR measurements lead Flasar et al. (2005) to infer a $\mathrm{C}$ enrichment of $8.8 \pm 1.7$ compared to solar from the fit of $\mathrm{CH}_{4}$ rotational lines.

Moreover, from an indirect determination, nitrogen has been shown to be enriched by a factor of $3.1 \pm 0.6$ (Briggs $\&$ Sackett 1989; ground-based radio wavelength observations) in Saturn's atmosphere compared to solar. Briggs \& Sacketts (1989) also estimated that $S$ is enriched by a factor of $\sim 12$ compared to solar in Saturn. However, this latter estimation seems to be uncertain due to the difficulty of distinguishing between the contribution to the opacity of $\mathrm{H}_{2} \mathrm{~S}$ from that of other compounds in the microwave spectrum (Hersant et al. 2004). For this reason, we assume in the present work that the abundance of $\mathrm{S}$ is still unknown.

\section{Formation of Saturn}

The model of Saturn's formation considered here is the one presented by A05b. In this model, Saturn forms from an embryo originally located at $\sim 12 \mathrm{AU}$. The proto-planet migrates inwards by following proto-Jupiter's trail and stops at the current

\footnotetext{
${ }^{1}$ Note that we use the central values of species abundances derived from Table 2 of Lodders (2003) in our calculations, contrary to A05b and $\mathrm{A} 05 \mathrm{c}$ who adopted a set of values within the error bars to obtain the best fit possible of the volatile enrichments in Jupiter.
} 
Table 1. List of measurements of $\mathrm{CH}_{4}$ abundances in Saturn's atmosphere published since 1981.

\begin{tabular}{cccc}
\hline \hline $\mathrm{CH}_{4} / \mathrm{H}_{2}\left(\times 10^{-3}\right)$ & Solar enrichment & Reference & Measurement \\
\hline $2 \pm 0.5$ & $3.4 \pm 0.9$ & Kerola et al. (1997) & KAO \\
$2_{-0.8}^{+1.0}$ & $3.4_{-1.4}^{+1.7}$ & Encrenaz \& Combes (1982) & ground-based \\
$2.2_{-0.2}^{+0.8}$ & $3.8_{-0.3}^{+1.4}$ & Killen (1988) & ground-based \\
2.5 & 4.3 & Tomasko \& Doose (1984) & Pioneer IPP \\
$3 \pm 0.6$ & $5.2 \pm 1.0$ & Karkoschka \& Tomasko (1992) & ground-based \\
$4 \pm 2$ & $6.9 \pm 3.4$ & Buriez \& de Bergh (1981) & ground-based \\
$4.2 \pm 0.4$ & $7.2 \pm 0.7$ & Trafton (1985) & ground-based \\
$4.4 \pm 1.2$ & $7.6 \pm 2.1$ & Lellouch et al. (2001) & ISO-SWS \\
$4.5_{-1.9}^{+2.4}$ & $7.7_{-3.3}^{+4.1}$ & Courtin et al. (1984) & Voyager IRIS \\
$5.1 \pm 1.0$ & $8.8 \pm 1.7$ & Flasar et al. (2005) & Cassini CIRS \\
\hline
\end{tabular}

position of Saturn when the disk disappears. Planetesimals are accreted during the whole formation process, along protoSaturn's migration pathway (between 12 and $9.8 \mathrm{AU}$ ). The resulting planet exhibits an internal structure comparable to that of the actual Saturn described by SG04 ${ }^{2}$, with a final core mass of $\sim 6 M_{\oplus}$ and a total mass of accreted planetesimals of $\sim 13.2 M_{\oplus}$. The thermodynamical conditions in the disk at the actual position of Saturn are used to calculate the composition of planetesimals that were accreted by the planet.

\section{Composition of planetesimals in Saturn's feeding zone}

In this work, we consider that abundances of all elements are solar and that $\mathrm{O}, \mathrm{C}$, and $\mathrm{N}$ exist only in the form of $\mathrm{H}_{2} \mathrm{O}$, $\mathrm{CO}_{2}, \mathrm{CO}, \mathrm{CH}_{4}, \mathrm{~N}_{2}$ and $\mathrm{NH}_{3}$ in the solar nebula gas-phase. Concerning the initial ratios of $\mathrm{CO}_{2}: \mathrm{CO}: \mathrm{CH}_{4}$ and $\mathrm{N}_{2}: \mathrm{NH}_{3}$ in the solar nebula, for the sake of consistency, we adopt the same range of values as the ones derived by A05c to fit the observed enrichments in volatiles in Jupiter. Hence, we assume $\mathrm{CO}_{2}: \mathrm{CO}: \mathrm{CH}_{4}=40: 10: 1$ to $10: 10: 1$ for $\mathrm{N}_{2}: \mathrm{NH}_{3}=1$ and $\mathrm{CO}_{2}: \mathrm{CO}: \mathrm{CH}_{4}=10: 10: 1$ for $\mathrm{N}_{2}: \mathrm{NH}_{3}=10$ in the vapor phase of the solar nebula. The considered values of $\mathrm{CO}_{2}: \mathrm{CO}: \mathrm{CH}_{4}$ are consistent with ISM measurements (Allamandola et al. 1999; Gibb et al. 2004). The adopted values of $\mathrm{N}_{2}: \mathrm{NH}_{3}$ are compatible with thermochemical calculations in the solar nebula that take into account catalytic effects of Fe grains on the kinetics of $\mathrm{N}_{2}$ to $\mathrm{NH}_{3}$ conversion (Fegley 2000). In addition, $\mathrm{S}$ is assumed to exist in the form of $\mathrm{H}_{2} \mathrm{~S}\left(\mathrm{H}_{2} \mathrm{~S} / \mathrm{H}_{2}=0.7 \times\left(\mathrm{S} / \mathrm{H}_{2}\right)_{\odot}\right.$, following $\mathrm{A} 05 \mathrm{c}$ ) and other sulfur compounds in the solar nebula (Pasek et al. 2005).

We also assume that volatiles have been trapped during the cooling of the nebula in planetesimals either in form of pure condensates, as it is the case for $\mathrm{CO}_{2}$, or in form of hydrates or clathrate hydrates, as it is the case for $\mathrm{NH}_{3}, \mathrm{~N}_{2}, \mathrm{CH}_{4}, \mathrm{CO}, \mathrm{Xe}$, $\mathrm{Ar}$ and $\mathrm{Kr}$ (see A05c for details). Note that the amount of available water ice is assumed to be sufficient to trap all volatiles

\footnotetext{
${ }^{2}$ Detailed internal structure models of Saturn developed by SG04 indicate a maximum mass of heavy elements present in the envelope between nearly 0 and $10 M_{\oplus}$, whereas the mass of the core varies between 8 and $25 M_{\oplus}$. However, the minimum mass of the solid core might be decreased by up to $\sim 7 M_{\oplus}$ depending upon the extent of sedimented helium.
}

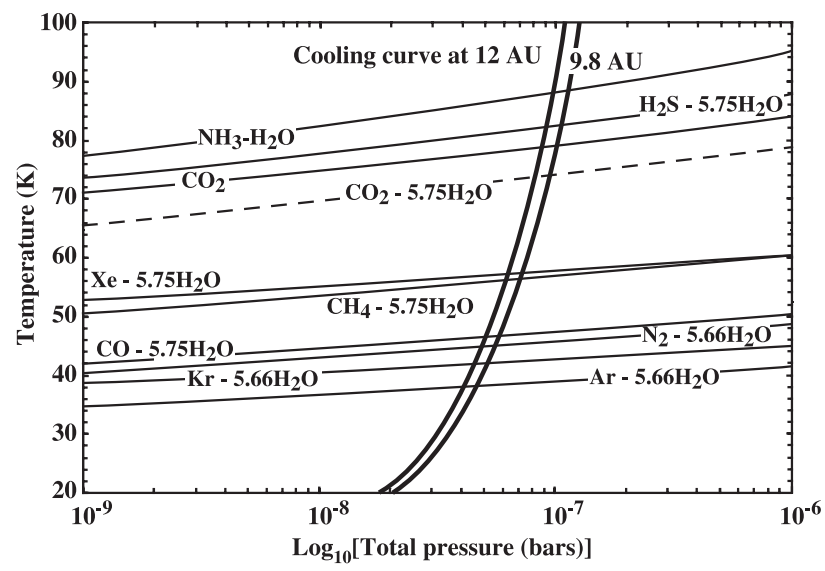

Fig. 1. Stability curves of the condensates considered in the present work, and evolutionary tracks of the nebula at 12 and $9.8 \mathrm{AU}$. Abundances of various elements are solar, with $\mathrm{CO}_{2}: \mathrm{CO}: \mathrm{CH}_{4}=$ 30:10:1 and $\mathrm{N}_{2}: \mathrm{NH}_{3}=1$ in vapor phase. The condensation curve of $\mathrm{CO}_{2}$ pure condensate (solid line) is shown together with that of the corresponding clathrate hydrate (dashed line). Species remain in the vapor phase as long as they stay in the domains located above the curves of stability.

except $\mathrm{CO}_{2}$. Once condensed, ices are assumed to decouple from the gas and to be incorporated into growing planetesimals which may subsequently be accreted by the forming Saturn.

Figure 1 represents the cooling curves of the nebula at 9.8 and $12 \mathrm{AU}$ derived from the solar nebula model used by A05b, as well as the condensation curves for the various ices considered in this work. The stability curves of clathrate hydrates are derived from Lunine \& Stevenson (1985) whereas we used a fit to the experimental data for the pure $\mathrm{CO}_{2}$ condensate (Lide 1999). Figure 1 provides the condensation sequence of the different volatiles initially existing in vapor phase inside Saturn's feeding zone. The intersection between the cooling curve and the stability curve of the different condensates also gives the thermodynamic conditions at which the different ices are formed.

Using Eqs. (1) and (2) given in A05c, we can calculate 1) the minimum abundance of water required to trap all volatiles (except $\mathrm{CO}_{2}$ ) in the solar nebula gas-phase to form clathrate hydrates or hydrates and 2) the composition of ices incorporated in planetesimals that were accreted by proto-Saturn. 
Table 2. Gas phase abundances (molar mixing ratio with respect to $\mathrm{H}_{2}$ ) of major species in the solar nebula (from Lodders 2003) for $\mathrm{CO}_{2}: \mathrm{CO}: \mathrm{CH}_{4}=30: 10: 1$ and $\mathrm{N}_{2}: \mathrm{NH}_{3}=1$.

\begin{tabular}{lclc}
\hline \hline Species $i$ & \multicolumn{1}{c}{$x_{i}$} & Species $i$ & $x_{i}$ \\
\hline $\mathrm{O}$ & $1.16 \times 10^{-3}$ & $\mathrm{~N}_{2}$ & $5.33 \times 10^{-5}$ \\
$\mathrm{C}$ & $5.82 \times 10^{-4}$ & $\mathrm{NH}_{3}$ & $5.33 \times 10^{-5}$ \\
$\mathrm{~N}$ & $1.60 \times 10^{-4}$ & $\mathrm{~S}$ & $3.66 \times 10^{-5}$ \\
$\mathrm{H}_{2} \mathrm{O}$ & $1.66 \times 10^{-4}$ & $\mathrm{Ar}$ & $8.43 \times 10^{-6}$ \\
$\mathrm{CO}_{2}$ & $4.26 \times 10^{-4}$ & $\mathrm{Kr}$ & $4.54 \times 10^{-9}$ \\
$\mathrm{CO}$ & $1.42 \times 10^{-4}$ & $\mathrm{Xe}$ & $4.44 \times 10^{-10}$ \\
$\mathrm{CH}_{4}$ & $1.42 \times 10^{-5}$ & & \\
\hline
\end{tabular}

Table 3. Calculations of the ratios of trapped masses of volatiles to the mass of $\mathrm{H}_{2} \mathrm{O}$ ice in planetesimals formed at 9.8 and $12 \mathrm{AU}$ in the solar nebula. Gas-phase abundance of $\mathrm{H}_{2} \mathrm{O}$ is equal to $8.11 \times 10^{-4}$ ( $\sim 4.9$ times the value quoted in Table 2 ; see text), and gas-phase abundances of elements are assumed to be solar (Lodders 2003) with $\mathrm{CO}_{2}: \mathrm{CO}: \mathrm{CH}_{4}=30: 10: 1$ and with $\mathrm{N}_{2}: \mathrm{NH}_{3}=1$ in vapor phase in the solar nebula. The abundance of $\mathrm{H}_{2} \mathrm{~S}$ is subsolar (see text).

\begin{tabular}{lcc}
\hline \hline & $9.8 \mathrm{AU}$ & $12 \mathrm{AU}$ \\
\hline $\mathrm{CO}_{2}: \mathrm{H}_{2} \mathrm{O}$ & $8.91 \times 10^{-1}$ & $9.13 \times 10^{-1}$ \\
$\mathrm{CO}: \mathrm{H}_{2} \mathrm{O}$ & $1.46 \times 10^{-1}$ & $1.45 \times 10^{-1}$ \\
$\mathrm{CH}_{4}: \mathrm{H}_{2} \mathrm{O}$ & $9.14 \times 10^{-3}$ & $9.23 \times 10^{-3}$ \\
$\mathrm{~N}_{2}: \mathrm{H}_{2} \mathrm{O}$ & $5.26 \times 10^{-2}$ & $5.34 \times 10^{-2}$ \\
$\mathrm{NH}_{3}: \mathrm{H}_{2} \mathrm{O}$ & $4.67 \times 10^{-2}$ & $4.66 \times 10^{-2}$ \\
$\mathrm{H}_{2} \mathrm{~S}: \mathrm{H}_{2} \mathrm{O}$ & $4.28 \times 10^{-2}$ & $4.32 \times 10^{-2}$ \\
$\mathrm{Ar}: \mathrm{H}_{2} \mathrm{O}$ & $9.74 \times 10^{-3}$ & $9.94 \times 10^{-3}$ \\
$\mathrm{Kr}: \mathrm{H}_{2} \mathrm{O}$ & $1.34 \times 10^{-5}$ & $1.34 \times 10^{-5}$ \\
$\mathrm{Xe}: \mathrm{H}_{2} \mathrm{O}$ & $2.44 \times 10^{-6}$ & $2.45 \times 10^{-6}$ \\
\hline
\end{tabular}

Since, as shown in Table 3, the composition of planetesimals does not vary significantly along the migration path of Saturn, we assume that all the ices contained in accreted planetesimals have an identical composition.

\section{Enrichments in volatiles in Saturn}

From the determination of the composition of the ices incorporated in the planetesimals (which depend on the initial gasphase composition in the solar nebula) and the assumption of a mean $I / R$ value for the accreted planetesimals, it is possible to calculate the expected enrichments in volatiles in Jupiter's atmosphere. In Table 4 we present the results of these computations and compare them to the observed enrichments for $\mathrm{CO}_{2}: \mathrm{CO}: \mathrm{CH}_{4}=30: 10: 1$ and $\mathrm{N}_{2}: \mathrm{NH}_{3}=1$ (our nominal vapor phase conditions) in the solar nebula gas-phase. Our model reproduces the $\mathrm{C}$ and $\mathrm{N}$ ground-based enrichment measurements. The minimum fit of the airborne $\mathrm{C}$ and ground-based $\mathrm{N}$ measurements corresponds to an $\mathrm{O}$ enrichment of 5.4 times the solar value. From this, we predict that $\mathrm{S}, \mathrm{Ar}, \mathrm{Kr}$ and $\mathrm{Xe}$ are overabundant compared to solar by a factor of 2.1, 2.0, 2.2 and 2.6 respectively. In addition, these enrichments result from the accretion of $5.6 M_{\oplus}$ of ices including $2.5 M_{\oplus}$ of water in the
Table 4. Observed $\mathrm{C}$ and $\mathrm{N}$ enrichments in Saturn, and calculated enrichments in volatiles in the nominal model $\left(\mathrm{CO}_{2}: \mathrm{CO}: \mathrm{CH}_{4}=30: 10: 1\right.$ and $\mathrm{N}_{2}: \mathrm{NH}_{3}=1$ ).

\begin{tabular}{|c|c|c|c|c|}
\hline \multirow[t]{2}{*}{ Species } & \multicolumn{2}{|c|}{ Observed } & \multicolumn{2}{|c|}{ Calculated } \\
\hline & (1) & (2) & (1) & (2) \\
\hline $\mathrm{C}$ & $3.4 \pm 0.9^{a}$ & $8.8 \pm 1.7^{b}$ & 2.8 & 7.1 \\
\hline $\mathrm{N}$ & \multicolumn{2}{|c|}{$3.1 \pm 0.6^{c}$} & 2.5 & 6.5 \\
\hline S & & & 2.1 & 5.5 \\
\hline $\mathrm{Ar}$ & & & 2.0 & 5.1 \\
\hline $\mathrm{Kr}$ & & & 2.2 & 5.6 \\
\hline $\mathrm{Xe}$ & & & 2.6 & 6.7 \\
\hline
\end{tabular}

The observed values are taken from ${ }^{a}$ Kerola et al. (1997); ${ }^{b}$ Flasar et al. (2005); and ${ }^{c}$ Briggs \& Sackett (1989). Calculated enrichments are calibrated on the observed $\mathrm{C}$ enrichment with the same column label.

envelope of Saturn. The resulting total content in heavy elements in Saturn is therefore compatible with the formation model of A05b and consequently with the internal structure model of SG04, provided the ratio of ices to rocks $(I / R)$ is greater than or equal to $\sim 0.7$. Table 5 summarizes the ranges of required masses of ices in the envelope of Saturn, and the resulting $\mathrm{O}$ enrichments, to match both $\mathrm{C}$ and $\mathrm{N}$ ground-based measurements if the initial gas phase conditions are varied. It can be seen that $O$ is enriched at least between 5.1 and 7.6 times the solar abundance in Saturn's atmosphere while the minimum mass of incorporated ices varies between 5.4 and $7.6 M_{\oplus}$ in the giant planet. Under these conditions, the minimum $I / R$ ratio required in Saturn varies between $\sim 0.7$ and 1.4 in order to be compatible both with the formation model of A05b and the internal structure model of SG04.

On the other hand, fitting the recent $\mathrm{C}$ measurement derived from Cassini data does not allow one to retrieve a value of $\mathrm{N}$ compatible with the measured one. In the nominal case, fitting the minimum value of the Cassini measurement implies overabundances compared to solar of N, S, Ar, Kr and Xe by factors of $6.5,5.5,5.1,5.6$ and 6.7 respectively. These calculations translate to an $\mathrm{O}$ enrichment of at least 13.9 times the solar value in the planet's envelope and require the accretion of at least $14.4 M_{\oplus}$ of ices including $6.6 M_{\oplus}$ of water. Following Table 5, if the initial gas-phase conditions are modified, the minimum mass of accreted ices varies between 13.7 and $18.3 M_{\oplus}$, whereas the corresponding $\mathrm{O}$ enrichment ranges between 13.1 and 18.3 times the solar value in the giant planet's atmosphere. In all these cases, the minimum amount of required ices exceeds the mass of heavy elements accreted by the Saturn's formation model of A05b. Moreover, this minimum mass also exceeds the mass of heavy elements present in the planet's atmosphere derived by SG04 (of the order of $\sim 10 M_{\oplus}$ ). However, it is still compatible with the global amount of heavy elements predicted by SG04 $\left(\leq 30 M_{\oplus}\right)$. 
Table 5. Minimum mass of accreted water $\left(M_{\text {water }}\right)$, minimum mass of accreted ices $\left(M_{\text {ices }}\right)$ and corresponding minimum $\mathrm{O} / \mathrm{H}$ abundance (compared to solar value) in Saturn's atmosphere required to fit the observed C enrichments. Calculations are presented for the minimum fits of both KAO C and ground-based N measurements (Kerola et al. 1997; Briggs \& Sackett 1989) and Cassini C measurement (Flasar et al. 2005) in Saturn's atmosphere. Ranges of $\mathrm{CO}_{2}: \mathrm{CO}: \mathrm{CH}_{4}$ and $\mathrm{N}_{2}: \mathrm{NH}_{3}$ gas-phase ratios used here are those determined by A05c (see text).

\begin{tabular}{|c|c|c|c|c|c|c|}
\hline & & \multicolumn{4}{|c|}{$\mathrm{N}_{2}: \mathrm{NH}_{3}=1$} & \multirow{2}{*}{$\frac{\mathrm{N}_{2}: \mathrm{NH}_{3}=10}{\mathrm{CO}_{2}: \mathrm{CO}=1}$} \\
\hline & & $\mathrm{CO}_{2}: \mathrm{CO}=4$ & $\mathrm{CO}_{2}: \mathrm{CO}=3$ & $\mathrm{CO}_{2}: \mathrm{CO}=2$ & $\mathrm{CO}_{2}: \mathrm{CO}=1$ & \\
\hline \multirow[t]{3}{*}{ KAO } & $M_{\text {water }} / M_{\oplus}$ & 2.2 & 2.5 & 3.0 & 4.0 & 4.6 \\
\hline & $M_{\mathrm{ices}} / M_{\oplus}$ & 5.4 & 5.6 & 5.9 & 6.6 & 7.6 \\
\hline & $\mathrm{O} / \mathrm{H}$ & 5.1 & 5.4 & 5.8 & 6.6 & 7.6 \\
\hline \multirow[t]{3}{*}{ Cassini } & $M_{\text {water }} / M_{\oplus}$ & 5.7 & 6.6 & 8.0 & 11.0 & 11.2 \\
\hline & $M_{\mathrm{ices}} / M_{\oplus}$ & 13.7 & 14.4 & 15.7 & 18.2 & 18.3 \\
\hline & $\mathrm{O} / \mathrm{H}$ & 13.1 & 13.9 & 15.2 & 18.0 & 18.3 \\
\hline
\end{tabular}

\section{Discussion and conclusions}

In the framework of the clathrate hydrates trapping theory and using the gas-phase abundances of major volatile species in the solar nebula used by A05c to fit the volatile enrichments in Jupiter, we have calculated the enrichments in O, C, N, S, Xe, $\mathrm{Ar}$, and $\mathrm{Kr}$ with respect to their solar abundances in the atmosphere of Saturn. Two different measurements of the abundance of carbon in Saturn have been considered for the calibration of our calculations, namely the ground-based determination derived by Kerola et al. (1997) and the recent Cassini measurement as published by Flasar et al. (2005).

Using the near-IR determination of $\mathrm{C}$ by Kerola et al. (1997), we are able to fit the measured value of $\mathrm{N}$ enrichment. Moreover, the predicted amount of ices accreted by Saturn is consistent with the formation model of A05b and the internal structure model of SG04, provided the minimum $I / R$ ratio required in the giant planet varies between $\sim 0.7$ and 1.4 , a value compatible with the recent Cassini determination of Phoebe's low density (1630 $\mathrm{kg} \mathrm{m}^{-3}$; Porco et al. 2005).

On the other hand, using the recent far-IR determination of $\mathrm{C}$ reported by Flasar et al. (2005), the measured value of $\mathrm{N}$ enrichment could no longer be reproduced. In addition, the amount of ices accreted by Saturn exceeds the mass of heavy elements predicted by the A05b model. This difference is likely to be due to the assumptions made by A05b in the calculation of the planetesimal's disk (see also Alibert et al. 2005a, for details).

Furthermore, the amount of heavy elements required to explain the Cassini measurement that should be currently in Saturn's envelope appears incompatible with the results of the internal structure modeling by SG04 (Table 5). In addition, in order to explain only the value of $\mathrm{C}$ enrichment given by Flasar et al. (2005), one needs to invoke the accretion of at least $10.8 M_{\oplus}$ of ices (including a minimum of $3.9 M_{\oplus}$ of water) in the envelope of Saturn with our nominal gas-phase conditions, an amount slightly exceeding the maximum mass of heavy elements predicted in this zone by SG04. The discrepancy is higher if one adds the amount of ices required to trap $\mathrm{N}_{2}$ and $\mathrm{NH}_{3}$ as clathrate hydrates or hydrates in planetesimals: the measured abondance of N by Briggs \& Sackett (1989) requires as a minimum the additional accretion of $0.9 M_{\oplus}$ of ices, whereas assuming an enrichment of $\mathrm{N}$ of 6.5 (as predicted by our calculations) requires the accretion of a minimum of $2.3 M_{\oplus}$ of ices. Therefore, in order to reconcile Saturn's internal structure models and the $\mathrm{C}$ measurement made by the Cassini spacecraft, one must then suppose a sedimentation of some of the accreted water onto the core, although the major part of other accreted volatiles would have remained in the atmosphere. This assumption is compatible with the work of Fortney \& Hubbard $(2003,2004)$ who argued that a significant oxygen depletion may have occurred in Saturn's atmosphere during its thermal history. This may be due to the immiscibility of oxygen in hydrogen at high temperature and pressure conditions (Fortney \& Hubbard 2003).

A number of ground-based $\mathrm{C}$ measurements such as those of Buriez \& de Bergh (1981) and Trafton (1985) are in agreement with the Cassini CIRS data, along with the Voyager IRIS data utilized by Courtin et al. (1984) and the ISO-SWS determination by Lellouch et al. (2001; see Table 1). This implies that the Cassini determination would be more representative of the carbon abundance in Saturn's atmosphere than that of Kerola et al. (1997). On this basis, we note that our global oxygen abundance in Saturn is at least $\sim$ two times higher than that predicted by Visscher \& Fegley (2005) from net thermochemical reaction calculations in its atmosphere. On the other hand, the calculations of Visscher \& Fegley (2005) are consistent with the current Saturn's atmospheric composition and may not be valid for the whole planet.

Acknowledgements. This work was supported in part by the Swiss National Science Foundation. We thank Jean-Marc Petit and Emmanuel Lellouch for helpful remarks.

\section{References}

Alibert, Y., Mordasini, C., \& Benz, W. 2004, A\&A, 417, L25

Alibert, Y., Mordasini, C., Benz, W., et al. 2005a, A\&A, 434, 343

Alibert, Y., Mousis, O., Mordasini, C., et al. 2005b, ApJ, 626, L57 (A05b)

Alibert, Y., Mousis, O., \& Benz, W. 2005c, ApJ, 622, L145 (A05c)

Allamandola, L. J., Bernstein, M. P., Sandford, S. A., et al. 1999, Space Sci. Rev., 90, 219

Briggs, F. H., \& Sackett, P. D. 1989, Icarus, 80, 77 
Buriez, J. C., \& de Bergh, C. 1981, A\&A, 94, 382

Courtin, R., Gautier, D., Marten, A., et al. 1984, ApJ, 287, 899

Encrenaz, T., \& Combes, M. 1982, Icarus, 52, 54

Fegley, B. Jr. 2000, Space Sci. Rev., 92, 177

Flasar, F. M., Achterberg, R. K., Conrath, B. J., et al. 2005, Science, 307,1247

Fortney, J. J., \& Hubbard, W. B. 2003, Icarus, 164, 228

Fortney, J. J., \& Hubbard, W. B. 2004, ApJ, 608, 1039

Gibb, E. L., Whittet, D. C. B., Boogert, A. C. A., \& Tielens, A. G. G. M. 2004, ApJS, 151, 35

Hersant, F., Gautier, D., \& Lunine, J. I. 2004, Planet. Space Sci., 52, 623

Karkoschka, E., \& Tomasko, M. G. 1992, Icarus, 97, 161

Kerola, D. X., Larson, H. P., \& Tomasko, M. G. 1997, Icarus, 127, 190
Killen, R. M. 1988, Icarus, 73, 227

Lellouch, E., Bézard, B., Fouchet, T., et al. 2001, A\&A, 670, 610 Lide, D. R. 1999, CRC Handbook of Chemistry and Physics, ed. D. R. Lide (Boca Raton: CRC press LLC), 6

Lodders, K. 2003, ApJ, 591, 1220

Lunine, J. I., \& Stevenson, D. J. 1985, ApJS, 58, 493

Pasek, M. A., Milsom, J. A., Ciesla, F. J., et al. 2005, Icarus, 175, 1

Pollack, J. B., Hubickyj, O., Bodenheimer, P., et al. 1996, Icarus, 124, 62

Porco, C. C., Baker, E., Barbara, J., et al. 2005, Science, 307, 1237

Saumon, D., \& Guillot, T. 2004, ApJ, 609, 1170 (SG04)

Tomasko, M. G., \& Doose, L. R. 1984, Icarus, 58, 1

Trafton, L. 1985, Icarus, 63, 374

Visscher, C., \& Fegley, B. 2005, ApJ, 623, 1221 